\title{
Virtual Worlds: Relationship Between Real Life and Experience in Second Life
}

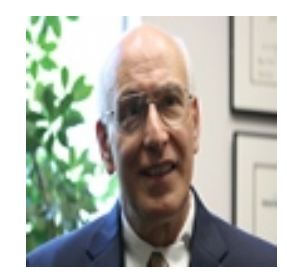

Scott P. Anstadt, Shannon Bradley, Ashley Burnette, and Lesley L. Medley Florida Gulf Coast University, USA

\section{Abstract}

Due to the unique applications of virtual reality in many modern contexts, Second Life (SL) offers inimitable opportunities for research and exploration and experiential learning as part of a distance learning curriculum assignment. A review of current research regarding SL examined real world social influences in online interactions and what the effects on users may be. This aids students in understanding the social constructionist perceptions and worldview of those persons they may serve in social services. This suggests the importance of developing an understanding of the relationship between users' real life (RL) and their SL. Some research has begun to reveal the effectiveness of telecommunication and computer simulation with certain clients in the fields of mental health and social work, yet there is a lack of sufficient research done within the context of virtual worlds. The current study surveyed users of several educationally and health focused SIMS (simulations) as to what motivates their SL and RL interactions. The data explores associations between users' RL and their SL in several areas,potentially addressing the future role of educating social work students regarding research methodology in online virtual reality interactions. Implications for social work are discussed including engaging clients using incentives for social participation built into the SL milieu.

Keywords: Social work; virtual world; Second Life 


\section{Introduction}

Multi-user virtual environments (MUVEs) are perceptual and interactive simulated worlds in which persons might discover lifestyles, traditions, and engagement in interactive conversations in a role-playing medium. MUVEs afford access to ongoing social interaction, information both in print and depicted through video casts, and integration of collaborative learning through group sharing and teambuilding efforts (Dillenbourg, Schneider, \& Syneta, 2002). Unlike other forms of virtual education, MUVEs support synchronous communication, therefore approaching what might be seen as a learning discourse in a sense of community (Barab, Thomas, Dodge, Carteaux, \& Tuzun, 2005; Bruckman, 1997; DeLucia, Francese, Passero, \& Tottora, 2009). Major professional organizations in the field of educational technology, such as the New Media Consortium, the International Society for Technology in Education, and the Association for Educational Communications and Technology, actively use and study applications of MUVEs in educational programming.

As a well-known example of MUVEs, Second Life (SL) is used by over 200 educational institutions throughout the world. Developed by Linden Labs in 2003, SL estimates over 15 million users, and in any one point in time an average of about 50,000 (Baker, Wentz, \& Woods, 2009). Individuals create avatars as virtual representations of their real physical selves which serve to navigate the virtual simulators ("SIMS") in the form of humans, animals, inanimate objects, or hybrids known as "furries" (Bell, Castranova, \&Wagner, 2009, p. 23; Gottschalk, 2010).

As such, SL offers a rich 3D virtual world that provides an unprecedented context for research using a social constructionist learning theoretical framework within an "authentic" and "living" reality. Epitomizing social constructionist principles, users in SL have access to a vast array of tools and audiences for the creative construction of "world-building" (Aurilio, 2010; Dawley, 2009). Social constructionist theory contends that deeper knowledge structures are developed as people interact with their physical and social world and engage in building artifacts. The social construction of reality occurs as individuals and groups interact and this interaction is how social phenomena are created. Socially constructed reality is an ongoing, dynamic process, where culture intertwined with history is seen as the source of human thought and behavior. In social constructionist theory learning continuously occurs and cannot be separated from the social context, as the social context is at the center of meaning. The focus in social constructionist learning is on the artifacts or deeper knowledge created through the shared construction and collective generation and transmission of meaning through culture and history (Mutekwe, Ndofirepi, Maphosa, Wadesando, \& Machingambi, 2013; Mills, 2012; Conners, 2009; SparkNotes Editors, 2006; Brooks, 2002; Pinkett, 2000; Shaw, 1995; Vygotsky, 1978).

In recent years online communities, or SIMs, have been studied for their prospects in education. SL has been found to be a forum for tangibly enhancing quality of student learning by creating an experience which includes multi-sensory environments 
(J armon, Traphagan, \& Mayrath, 2008; Riedl, Bronack, \& Tashner, 2005; Squire \& Jenkins, 2003) and collaboration in student team projects (Erlandson, Nelson, \& Wilhelmina, 2010). SL features telling visual immersive components which helps students feel a greater sense of reality in the context of their learning. For this reason over 200 universities have a presence on SL. Here, classrooms in total can be run on SL and students may go out into the various SIMs for field trips, assimilated role-plays, and to gather information which can be experienced in both a visual and auditory manner. Therefore, the platform of SL can be incorporated into already established traditional classroom curriculum (Beltran, Sierra, Gutierrez, \& Garzon-Castro, 2012; Damianakis, Climans, \& Marziali,2009; Leonard, Withers, \& Sherblom, 2011; Rockinson-Szapkiw \& Walker, 2009). The context of education might include events which may exist in real life but are not as handily accessed. These include theater and visual arts, poetry, discussion groups, live entertainment, and self-help groups, among others.

In relation to distance learning and virtual educational environments, SL has garnered much attention among educationalists and researchers as a valuable learning platform for distance learning (Newman, Olle, \& Bradley, 2012; Anderson \& Dron, 2011; Aurilio, 2010; Sun, 2010; Dawley, 2009, p.113). Within the world of social work programs in higher education, SL has been increasingly integrated and utilized as an effective learning technology in distance education at University of Southern California, University of Georgia, Valdosta State University, Stanford University, East Carolina University, University at Buffalo, Indiana University, and University of Texas Arlington. Furthermore, current research in distance learning points to SL as providing an optimal framework for a virtual educational environment that exemplifies the embodiment of social constructionist principles (Mills, 2012; Aurilio, 2010; Conners, 2009). Additionally, SL provides an excellent platform for instructors to integrate scaffolding for students within their research, "as it is an amazing space for approaching research participants" (Conners, 2009, p. 103). Although educational institutions are increasingly including SL as a technology based tool in teaching and learning in distance education, there is a lack of research in guiding educators in course design and pedagogy within the SL environment (Mutekwe, Ndofirepi, Maphosa, Wadesando, \& Machingambi, 2013; Edwards, 2012; Jha, 2012; Sun, 2012; Anderson \& Dron, 2011; Conners, 2009; Dawley, 2009; Minocha \&Roberts, 2008; Huang, 2002).

Dawley (2009, p. 117) offers "social network knowledge construction" as an emerging pedagogical framework that addresses the need for new strategies in teaching and learning within distance learning courses in virtual worlds, such as SL. This framework incorporates user-centered content, social networking, and virtual worlds in the teaching repertoire that can be utilized in both inworld and out-of-world activities and in cross communication. The social construction of knowledge in the virtual world affords the students the opportunity to display physical representations of their newly gained knowledge in a variety of formats that can be shared with others and accessed at any time, further extending learning and deeper knowledge construction in a dynamic, ongoing process. 
For example, social work education has begun to make use of the virtual world format as a resource for achieving competence in the field. The Council on Social Work Education requires students to study human behavior in the social environment as well as assessment of clients using a biopsychsocial and spiritual format (Holloway, Black, Hoffman, \& Pierce, n.d.) . To this end there are full SIMs of communities in which the residents role-play a lifestyle consistent with ethnic, cultural, and spiritual traditions, thus bridging the gap between virtual lives and client/consumers' real lives (Anstadt, Burnette, \& Bradley, 2011,2012; Boelstorff, 2008; Eastwick \& Gardner, 2009; Parti, 2008).

Included are SIM representations of over 100 religious and spiritual traditions supporting a wide number of offerings in discussion groups, experiential expression of cultural traditions, and authentic cultural and artistic presentations. Avatars are assigned a name of their own and with a unique meaning to the puppeteer behind the keyboard. Role play of characters in the social context by the avatar residents allows the puppeteers to live these cultures authentically in this second life. Students may assume an anonymous avatar character and likewise experiment in involving themselves within various cultural environments on SL without feeling self-conscious as they reflect upon their experience. In this manner students feel more at ease in approaching educated informants on their experiences living within and practicing particular national, spiritual, and cultural traditions. Discussions with these resident informants allow students to reformulate and reconstruct their own impressions based upon information gleaned and comparison/contrast with their own background experiences. Unlike real life institutions, most spiritual and cultural settings in Second Life are free and open to anyone who wishes to participate (Baker, Wentz, \& Woods, 2009; Salmon, Nie, \& Edirisingha, 2010), thus allowing students easy access to many social contexts, interviews, and ethnographic observations (Boellstorff, Nardi, Pearce, \& Taylor, 2012)

Often, social workers and other human service workers face barriers to service which include distance from clients, time constraints, transportation, and access to services. The virtual world offers a potential solution to many of these barriers. For this reason, the coursework study of how SL and other virtual reality platforms may fit into social service delivery systems would be a fruitful way of developing virtual interventions within the applied field with clients. Budding social service students who participate in both the SL environment and in developing virtual field studies could add to the body of knowledge leading to the development of an array of social services which may be obtained through the distance platform. However, the vast expanse of the "metaverse" (internet universe of virtual reality) is still in its infancy, and research has just begun to explore the complex motivations and interactions of this rich virtual world.

Among the growing concepts that have been recently examined is that of the relationship between users' virtual lives and their RL qualities and characteristics. For instance, researchers in the field of virtual communications have questioned just how strong the influence of social mores, norms, and laws are in internet-based virtual realities such as SL (Boelstorff, 2008; Parti, 2008), what role accepted institutions, 
such as education, play in online interaction and learning (Nesson \& Nesson, 2008; Rockinson-Szapkiw \& Walker, 2009; Vernon, et. al., 2009), and what determines user acceptance and engagement in virtual constructs (Fetscherin \& Latteman, 2008; Tsan \& Day, 2007). Additional research has begun to determine the uses of virtual technologies in social services and continues to measure the efficacy of utilizing this new medium to enhance interventions, service delivery, and social service education (McCarty \& Clancy, 2002; Smokowski \& Hartung, 2003; Vernon, et. al., 2009). It is through increasing knowledge of what educators themselves are seeking in SL while exploring the resources in SIMs devoted to the pursuit of academics and health related matters that social service students can examine the potential of SL as a forum for social networking and social resource management. This exploration process may then be used with clients who may otherwise be isolated from elements of a rich social interactive environment now at their fingertips in SL (Belisle \& Bodur, 2010; Eastwick \& Gardner, 2009; Gottschalk, 2010, p. 506; Isabella, 2007; Stalker, 2007). Understanding, or at least uncovering, the answers to these and other questions could help to determine the potential for using SL as an intervention tool for the helping professions and thus to be studied as an integral part of distance education.

Building on research questions proposed by previous studies in SL (Bell, et.al., 2009, p.73; Gottschalk, 2010), this research seeks to demonstrate how students of social services may design and use a method which examines if there are any initial associations between RL interests that bring users to educational and health related themed SL SIMs, what they seek and do once they are into SL, and the purpose it provides for them. Based on the social constructionist theory, the researchers queried selected participants' real lives and their SL experience using survey and interview collection. This study is therefore an example of an immersive research application in SL conducted by graduate social work students.

\section{Method}

Data collection conducted by the authors studying in the field of social work focused on both quantitative and qualitative data, and because few studies have been done which examine the associations between these particular variables, it was largely exploratory. The study employed the use of several technologies new to collecting data in social work research and attempted to stay true to the nature of social work through direct interviews using a completely unique medium-SL itself. Two of the authors were MSW graduate students in social work at a university in the southeastern U.S. under the mentorship of the instructor of a required research methods course.

Initial approval was obtained via an expedited review from the Internal Review Board before beginning data collection. Participants were comprised of SL users who were most likely to frequent educational SIMs, such as those run by universities. For the purposes of this study, it was presumed that because SL requires users to be over the 
age of 18 to register, all participants were of legal age. Participants were users of SL who voluntarily selected to take the survey. Raw data was processed into SPSS and results analyzed using descriptive frequencies and crosstabs.

The qualitative survey was comprised of 37 questions related to the demographic information of the users and the purpose and quality of their activities and interactions in RL and SL (Appendix A). The survey was created for the purpose of this study and consultation on the key areas of interest which survey questions were intended to gather was elicited from experienced users of SL, professors of education, health care, and social work from within SL, as well as potential consumers of the survey who had some knowledge of online virtual reality prior to the study. From the feedback, changes to the survey were made to include additional demographic information and to rearrange questions for ease of understanding and to avoid potential bias.

An additional qualitative component to the study was added in order to expand upon the knowledge gained from the survey questions. The decision was made to enhance findings secured from the quantitative data with the use of interviews of self-selected participants. It was thought formative impressions gleaned would be useful in future revisions of the survey instrument. This information was collected through a semistructured, nine-question interview and discussion between the researchers' avatars and participants' avatars recorded in typed form (see Appendix B).

Distribution of the survey was controlled using an electronic "kiosk" which resembled a mailbox and which could be locked into place on SL SIMs. Initially, agreements were obtained from owners of educational and health oriented SIMs willing to allow kiosks to be placed on their virtual property. The owners of the SIMs consisted of SL presence of three universities which also existed in RL plus two that were exclusive to SL, two healthcare organizations in RL with a presence in SL, a hospice in SL, plus a SIM dedicated to accommodation services in both RL and SL for persons handicapped. Owners of the SIMs were given copies of the consent form and survey and were strongly encouraged not to coerce user participation in the survey at any time the survey was available on their property in order to preserve the voluntary nature of the study. The kiosks were placed in an agreed upon location on each SIM, with a total of nine SIM locations and were available for a period of eight months. Because of the electronic and computer-based nature of SL and the survey kiosks, computer "hacking" of the kiosks, which might compromise the information by uncovering the usernames and survey responses of participants, was a concern with the researchers as well as the SIM owners. To ensure participant confidentiality, the security of the kiosks was validated by the kiosk creator as well as tested and approved by the researchers and several of the SIM owners.

Occasionally due to system-wide changes, the kiosks were affected or became "glitchy", so the kiosks were checked weekly and replaced if necessary. As soon as they were recovered from the kiosks, each survey was coded and securely stored. At the end of one 
year, the researchers will no longer have any access to the raw data. After three years the raw data must be destroyed by the faculty advisor.

Once placed and secured, kiosks in each educational SIM in SL could be approached voluntarily by any avatar. Once they approached the kiosk, users were presented with an initial electronic prompt, encouraging them to accept a folder of information containing the consent form and study synopsis. Users were assured of the confidentiality of their responses and avatar usernames were not recorded. After reading the consent form, users were prompted to indicate their consent by responding "yes" to the first question of the survey. By indicating that they would like to continue past the consent form to the survey it was assumed that participants were giving their consent to participate. Users who responded "no" were directed away from the study and exited from the kiosk. Survey responses were sent immediately after completion to a secure email database.

Participants who agreed to take the survey were informed via the consent that they could exit the survey at any time, and all questions in the survey offered an "Exit" option which would navigate users away from the survey. Surveys which were unfinished were not included in the data collection process. After completing the survey, participants were again presented with another electronic prompt, thanking them for their participation and "giving" them a folder containing a gift of a free t-shirt for their avatar and instructions for how to contact the researchers for additional information about the study. Users were also informed about the voluntary qualitative interview portion of the survey, and were encouraged to contact the researchers to indicate their interest. Participants who declined to participate in the qualitative portion were not contacted by the researchers at any time after completion of the survey.

Since the interviews were only conducted after persons who took the survey consented by clicking on the last question on the survey, all who were interviewed came from the pool of persons who completed the survey. We had 11 interviews or $11 \%$ of the population who completed the survey. Any user interested in participating in the qualitative section contacted researchers via secure email and provided their avatar username and email address. Participants were then emailed the interview questions. Any participant who indicated they were comfortable answering the qualitative questions was considered to have given consent to the interview and was scheduled an avatar-to-avatar meeting in SL. Avatar usernames during the qualitative interviews were coded and were not connected to their survey answers to maintain confidentiality. The thematic analysis was done by a panel of four graduate students who had not been previously involved in the research. Each identified key themes that ran through the 11 interviews for each question. These themes were then compiled by them and presented to the researchers. 


\section{Results}

Demographic data was compiled and shows the breakdown of respondents as to RL age, ethnicity, gender, education, marital status, and residence (Appendix C). SL data shows the reported number of avatars, avatar age, and avatar gender.

Of the 100 completed survey participants, the largest number were between ages 31-50, followed by the next highest age group up to age 65. Over three quarters of the participants were Caucasian. This participant sample, which was gathered on predominantly educational and health related SIMs, was highly educated with 95\% having at least some college and $44 \%$ with a terminal graduate degree. More than two thirds came from the USA with the next highest contingent from Europe. In SL gender identification seemed to match identification in RL with most participants being female and a small percentage identified as 'other'. Relationship status in RL revealed that about half of the population surveyed were married; the next largest percentages were single (28\%) and divorced (12\%). Almost two thirds (62\%) of the completed surveys came from the SIM addressing physical and virtual handicaps. An additional 33\% came from the educational institutions and the remainder from the hospice and healthcare organizations. Many of these persons may frequent more than one of these SIMs.

In SL there is the freedom to be several identities by way of a number of avatars. A little over one third of participants indicated they had only one avatar, one quarter had two, and about one third had three or more. Of the participants $73 \%$ reported that they were using their first avatar to take the survey. Selected and informative associations between variable attributes were discovered when doing crosstabs as reported below. Due to the limited sample size and the non-parametric nature of the data, the data is reported in a descriptive manner below with additional analysis being left to future more robust study. Additional demographic information could be gleaned and organized from a representative sample of avatars in SL surveyed, which would be much more robust than reported here. Such a cross sampling may require extensive placements of kiosks or other methods of inworld survey distribution.

The following reported quantitative data address the research question showing trends in demographics and usage of those within our limited example who completed the survey, why they came to use SL, how they used SL, and to what purpose.

\section{Use of SL}

In response to the survey question of why participants first came to use SL, $46 \%$ said they were curious, followed by $13 \%$ meeting people, and $11 \%$ for educational purposes. In terms of length of calendar time on SL, almost half (49\%) reported 3-6 years, with the next highest number 19\% reporting 1-3 years, followed by $15 \%$ new persons who were on only under three months. Close to two thirds (61\%) reported signing into SL at least once per day with $33 \%$ signing in between 2-4 times per day. A full $62 \%$ of the participants spent at least 5 hours per week on SL with 17\% spending more than 20 
hours per week. Of those reporting (a) curiosity or (b) meeting people as their initial reasons for coming to SL, there was a high concentration of spending more than 10 hours per week on SL ([a] 54\% and [b] 49\% respectively). Over one third of participants (38\%) established what they believed to be a close relationship with another avatar on SL. Those committed to a marital or other significant relationship in RL (51\%) also tended to be involved in a significant relationship in SL (80\%) and to cluster in the 10 or more hours per week (56\%) of SL use. Of this group that reported being in a relationship, $24 \%$ responded that they were in a relationship longer than nine months, with $14 \%$ who reported relationship length as less than nine but more than three months. Of those relationships considered significant by the respondents, $77 \%$ were considered intimate.

Frequency of planned interpersonal contacts on SL and RL showed similar percentage rates on the low end of the scale with about one third (31\%) of the participants indicating they have less than three planned interpersonal contacts per week in both RL and SL. Of the one third (32\%) of participants who stated they have less than three social contacts per week in RL, 100\% said they had at least one social contact on SL per day. One quarter (26\%) of participants who have one or less RL planned interactions a day indicated they have at least five or more SL interactions.

\section{Purpose of SL}

Sixty percent of participants indicate belief that SL avatars represent in part the real person (puppeteer). Of those persons who came onto SL out of curiosity, $61 \%$ fell into this category. Of these participants who see the avatar as the representation of the real person, almost three quarters (73\%) indicated they could learn more about people because of the anonymity of the avatar. Of the total participants, almost half (46\%) engage in or believe all of SL is role play. Eighty-eight percent reported friendships were readily available in SL through such activities as dancing (62\%) and role plays (59\%), and a variety of groups (100\%), among others. Seventy-nine percent reported doing activities that they enjoyed currently or at one time in RL. In terms of what participants sought from the SL experience, $27 \%$ were in SL to have fun, $21 \%$ to build and create, $14 \%$ to meet people, and $12 \%$ for educational purposes. Of the three quarters (74\%) of this selected subpopulation who indicated they attended SL groups, educational groups at $33 \%$ and social groups at $18 \%$ were the top two selected. Sixty-five percent of all participants rated themselves as obtaining satisfaction in RL social relationships corresponding to $55 \%$ in SL relationships.

\section{Qualitative Analysis}

The following reported qualitative interview data address the research question showing more in depth thematic analysis within our limited example of why they came to use SL, how they used SL as a major and necessary social interaction vehicle, and the relationship between RL and SL roles. 
Of the 11 self-selected participants who volunteered to be interviewed, 8 came from the SIM addressing physical and virtual handicaps. A more precise sampling method to assure that this group was a representative sample of the larger group who completed the survey was not possible as the survey asked for volunteers and with respect to the participant's privacy considerations did not reach out to specific survey participants. Only those who agreed to be interviewed were contacted. A thematic analysis of qualitative interviews with participants revealed that these particular users' motivations for joining SL were for social support or interpersonal interactions of some kind, which they were not able to experience in $R L$ due to a physical disability or social impairment. Participants interviewed reported their motivations to participate in SL activities were influenced by the amount of time and the types of groups they engaged in, as well as the wide availability of groups in SL which were not available to them in RL due to these social or physical impairments.

These activities included development and attending self-help and other support groups, maintaining a small retail business, attending educational groups, social interaction, art projects, attending cultural activities, exploring new places, meeting new people, personal improvements in appearance and health, and participation in civic causes.

Engaging in role play activities occurs in a variety of forms, including the use of multiple avatars (alts) for diversified purposes. Typically, one avatar is the primary character but the other 'alts' have a particular function in particular settings. The alts are used to preserve the anonymity of the primary avatar. These alts are often used for role plays or for particular roles. Some examples include: gaming, teaching, alternative sexual practices, gender shifts, and alternative sexual identity. In most of the interviews, alts had long standing roles. The role plays were ongoing and had detailed story lines with a wide variety of social networks emerging and morphing within the role play setting.

Most of those interviewed had a significant other in RL but reported their additional relationships in SL did not serve as a threat to these RL relationships. Every person interviewed reported affiliation with at least one community in SL. Communities define themselves in terms of interpersonal interactions, culture, behavior standards, manner of communication, purpose in creating the ambience for a particular lifestyle, production of products to serve the public, and added anonymity to engage in certain practices. Most communities are open to visitors and some are closed.

Participants interviewed predominantly reported that their motivations for joining SL were either interest in social interactions, entertainment, or curiosity. Of those participants interviewed who reported having a disability, most reported that their need for socialization or stimulation was met in SL better than in RL, either due to isolation or physical impairment, and this positively influenced the amount of time spent engaging in SL communities and activities. 
Some specific examples include: parties and dances, sports, raising and breeding SL animals, community events, self-help groups, cultural events, art appreciation, philosophic debates, obtaining knowledge on a variety of useful subjects, philanthropic causes, building of all kinds including homes, communities, furniture, clothes, and so on.

The majority of interviewed participants (8 of 11) reported either a lack of RL social interactions due to physical limitation/isolation or a lack of ability to easily engage socially due to a social impairment. They all reported SL as a method of increasing their interpersonal interactions through interest groups, activities, communities, and friendships/relationships. SL was viewed by these participants as a way of increasing their socialization and/or practicing social interactions through the virtual medium. Interviewed participants most frequently identified that they could easily make friends and join interest groups in SL and were more engaged with these social interactions in SL than they were in RL. Time spent in SL was reported as being influenced by their engagement in SL and their lack of socialization in RL.

A smaller subset of the participants interviewed (2 of 11) reported that their participation in SL was a direct result of their activities in RL, which included work both physically and virtually on the educational SIMs. The interviews also supported a variety of options for SL gender representation including the use of asexual identities such as furries, and, on occasion, inanimate objects. However, this particular sample reported clear self-representation of gender between RL and SL gender.

\section{Discussion}

Because of the lack of time and staff members to explore and collect data from every or most SL communities, as there are hundreds, the sample size for this project is limited in scope. This is a potential limitation to the research, and for future research it is suggested that there be data collection throughout a wide variety of SL communities or many studies completed in a variety of locations to seek combined trends. This study represents the first step in this endeavor.

This exploratory study design yielded data from both quantitative (survey) and qualitative (interviews) sources. The two together help to put some formative solidity (for this limited sample) on the initial research question posed of why people seek out involvement in SL, what activities they frequent, and what purpose it serves for them. As is the case in many survey studies, much of the data was not clear and therefore qualitative impressions added a great deal to the understanding of the selections made by participants. Putting these two together leads to the following trends in this preliminary study. 
The quantitative and qualitative data suggest highly preferred activities in RL were also found to be motivating in a similar way in SL. About half of the respondents either role played or believed SL to be a world of role play. A large number of participants in all the RL relationship commitment categories in the survey reported that they believed they could make friends all or some of the time in SL, indicating that there is some positive feedback in interpersonal interactions or relationships in SL. As described in many of the interviews, such interactions become a RL motivator for $\mathrm{RL}$ social interactions and relationships. This ties into the predominant belief from survey results that persons on SL can get to know each other better due to the anonymity of the avatars. Note, however, that interviews came from educational and health sites. Communities with a different purpose and/ or culture may yield very different results.

Participant interviews indicate the degree to which SL interactions and activities fulfill needs that are not met in RL has an influence on the amount of time spent in SL. A segment of the respondents showed a limited (under 3 social contacts per week) social interaction in RL. Many of these persons at the same time indicated a much more frequent interaction per week on SL. Making friends in the context of key occupations including role-plays, building and creating, working at businesses, education, groups of all kinds, and exploring arts and music are some of the activities which occupy their time. In this sense, SL can be seen as an extension and representation of activities possible in RL but much more easily represented in SL. The qualitative interviews suggest that individuals who utilize SL to fulfill a need that is not met in RL due to physical impairment or social deficit may do so because of the lack of RL interactive opportunities. This is related to a key theme that dissatisfaction in interpersonal relationships in RL could be a motivator for individuals to join the virtual world for social interactions, communities of friends, or relationships.

This data suggests a strong social influence of RL on certain SL experiences, choices, and behaviors, for example, avatar gender, SL activities, SL interpersonal satisfaction, and SL relationships, as well as evidence that some social influences present in SL affect $\mathrm{RL}$ behavior, including the formation of relationships and time spent engaging in virtual interactions. However, as this study is largely exploratory in nature, additional data will need to be collected to determine directionality of influences as well as further relationships between RL and SL.

\section{Implications for Research and Limitations}

Recent research has developed a substantial base of understanding about many aspects of online virtual world use, including the cultural differences between SL and RL as well as the use of this technology for purposes such as marketing, anthropological research, and educational uses in online interactions (Bell et al., 2009; Eastwick \& Gardner, 2009; McCarty \& Clancy, 2002; Smokowski \& Hartung, 2003). However, there is a gap in the current research regarding what motivates users to join in the online melee and how the real world and the online world interact with each other. This is mainly in regards to how individuals function in interpersonal communications, as 
well as social influences exerted on the individual (Gottschalk, 2010 p. 511; Nesson \& Nesson, 2008).

SL provides individuals the opportunity to become someone other than who they are in RL. In essence, they can take on a new identity, leaving their RL identity behind. For many people this is liberating. In collecting data, avatar puppeteers are required to selfreport on the questionnaire. There is a question of how truthful their answers are, and which identity (avatar) they are portraying in their answers. Individuals on SL may have multiple avatars, thus allowing the same individual to take the survey multiple times and from several perspectives. In regards to honesty in self-report, this is an ongoing research issue even in $\mathrm{RL}$, which would require many factors to be taken into account. The current survey used asked about the puppeteer and not the avatars. To control for multiple uses, there would need to be some way of the puppeteers identifying themselves, which may risk confidentiality and further taint the data. In RL as well as SL participants may lie on their answers on a survey. On the other hand, a case could be made because SL is anonymous, individuals may choose to be more honest than they would in RL. Information on this important question may be obtained in additional survey studies gleaning information on how participants respond to SL surveys.

In the current research study, demographics such as age, gender, and ethnicity may have been influenced by the placement of the survey kiosks and the method of collection. Due to the placement of kiosks on educational and health care based SIMs, a bias in the participant age range (30-65 predominantly), gender (60/40 female to male ratio), and ethnicity (77\% Caucasian) as well as participant education (largely college, master's and doctoral degrees held) may exist and will need to be accounted for in future research. Therefore, some results, most especially related to reported ethnicity (analyzed with interactions in SL), were not included. The language of the survey (English) may have had an influence on this sample, possibly limiting the ethnicity of the participants taking the survey, so future studies utilizing this method of data collection may consider using a translated survey to acquire a more representative sample of SL participants.

This study did a form of content validity by having knowledgeable users review the survey. Additional studies might use surveys in a more narrowly defined SIM content, such as SIMs dedicated to specific lifestyles of participants. One example might be religious communities. This may allow for a greater potential of content and construct validity in developing the surveys. To allow for greatest flexibility in constructing the questions, a kiosk or other inworld distribution method should not have constraints on the length of questions. Reliability could be explored by administering the survey to SIMs previously used to see if similar results would be gleaned. Reliability could also be explored by retesting a sample of those who took the survey after a period of time has elapsed. However, many life circumstances both in SL and RL would affect the results. Over the course of studies a clear procedure for establishing inter-rater reliability of coding must also be established based upon the agreed upon definitions of emerging 
themes. Should inworld data collection be successful in a well-developed stepwise survey delivery as well as validity for particular SL population samples, this opens the doors for further and more pertinent research that is capable of maintaining the integrity of the unique socio-cultural atmosphere of SL and similar virtual worlds. This may become a key strength of the research in this area.

The method of collection revealed a significant influence on survey completion, as another 99 surveys engaged were "timed out" and incomplete, and therefore it was necessary for any data collected in these surveys to be excluded. Once timed out, the data was not forwarded to the database. In these cases it was only known that a survey was started but not completed.

Reasons for this may include the system-wide changes that SL experiences on a continual basis affecting the functionality of the kiosks, or a bias which eliminates users who are unfamiliar with how to use the kiosk and interferes with their ability to take the survey. Future use of the inworld collection method should explore additional technological resources for distributing surveys which are more reliable, more userfriendly, and again possibly multi-lingual.

Further limitations in the use of this particular kiosk included the limit to the number of characters which the kiosk could hold, so the surveys needed to be edited from 42 questions to 37 questions to accommodate the amount of memory space available in the kiosk. A total of 12 surveys taken were missing some data due to these kiosk limitations. It was also important to consider the limitations that exist in data collection in online virtual worlds, including concerns about maintaining participant confidentiality in a venue where computer hacking is somewhat prevalent (Bell et al., 2009, p. 42; McCarty $\&$ Clancy, 2002). The survey used also presented limitations as it was designed specifically for use in this study and is not a validated instrument; therefore it must be refined in future research in both form and function.

It was noted, however, that the inworld collection method of survey kiosks was an effective data collection tool in terms of appropriately securing survey information, and the kiosk was utilized consistently by SL participants. Therefore, future research in social services seeking to retain the social environment of SL during data collection should examine further options for exploring the use of SL survey kiosks. For example, placing kiosks on different types of SIMS, placing more kiosks, or refining the technology of the kiosks inworld could yield a more accurate and representative sample of the population within SL.

Also, research in the field of social services must adhere to a code of ethics and remain cognizant of the unique context of online virtual reality use. Further considerations must also be made for the future of research protocols, and the policies and ethical requirements of the Internal Review Board should take into account the quickly advancing technology of online virtual worlds. Given the unique technical potential of 
SL, this puts the responsibility on the researcher to take pains to be as ethical as possible when conducting research. Avatars may not be humans, but the person controlling and, more importantly, experiencing the avatar most certainly is, and therefore is susceptible to all of the vulnerabilities of more typical research subjects.

Should inworld data collection be successful, this opens the doors for further and more pertinent research which is capable of maintaining the integrity of the unique sociocultural atmosphere of SL and similar virtual worlds. This may become a key strength of the research in this area.

\section{Implications for Social Services Practice}

Technologically advanced research methods used inworld are improving the quality of the data collected and future advances in data collection can provide a more accurate representation of information (Bell, et. al, 2010) and begin to help human service workers uncover how services offered in SL might be applicable to their RL clients. Research in the field of social services is uniquely designed to examine how individuals and groups interact socially, and can be adapted to aid in the exploration of these interactions in SL and other similar online social venues in the fields of mental health counseling and social work education (Rockinson-Szapkiw \& Walker, 2009). In order to advance and bridge barriers to human services practice through the utilization of modern technology, there must first be an understanding of the nature of online worlds such as SL, the influences and relationships therein, and the potential for SL as a tool for social service interventions in the future.

With the information gleaned from this exploratory study, research in the field of social services can further expand on relevant knowledge about virtual worlds. From there we can begin to explore and predict what role human services practice has in the virtual realm, and whether it could be executed as an effective form of intervention and service delivery. For example, many young adults struggle to come to terms with their sexuality in a society where homosexuality and transgender assimilation is viewed with derision and condemnation. Their RL experiences might prompt them to join SL where using a "gender-swapped" avatar is completely appropriate and accepted. In turn these young adults can interact with other individuals with similar experiences and interests. This begs the question: Could the inception of an inworld support group directed by a social service worker be beneficial in creating a positive experience for this vulnerable population?

Results from the qualitative analysis provided some insight into the motivation for engaging in social relationships and activities in SL and the sense of community felt by many SL users. With many interviewees reporting that they were limited in RL by physical disabilities or social impairments/isolation, SL may provide a unique opportunity for them to reduce their level of isolation and improve their social supports and resources. As social isolation is a common condition for those in RL with mental health and physical health disorders, the community atmosphere available in SL could 
be a resource offered by social service workers to help their clients access therapeutic resources or even re-engage with much needed leisure activities and interests that may be lacking in their real lives. Professional human service workers could assist individuals with disabilities or their homebound caregivers by connecting them to social interactions and social support networks within SL without taxing them physically (Heron, Gentle, personal communication, April 29, 2011). This could reduce the impact of their disability or the disability of a loved one on their overall well-being.

Other groups that meet in SL, and even those individuals who have not yet discovered this online resource, could benefit from similar support systems. This includes individuals without transportation to an agency or facility, individuals with disabilities, individuals seeking a cultural experience, or those whose social interaction is limited due to personal illness or caregiver responsibilities. Using online virtual communication, human service workers could redefine service delivery by reaching a larger population of individuals and more diverse groups, and clients can access a broad network of social and educational support tools.

Social service workers may be able to use this tool to advance and broaden their scope of practice, bringing technology and education together. For instance, use of SL for practice courses in social work interventions enable educators the ability to utilize Web 2.0 technology, including the synchronous voice-chat and visual environment to target the Council on Social Work Education (CSWE) Educational Policy and Accreditation Standards (EPAS), such as professional identity and diversity, through experiential activities and role-play (Vernon, et. al, 2009). Interactions with both SL users as well as the educational communities within SL revealed just how diverse the cultural aspects and educational opportunities are in this virtual world.

In addition, special consideration must be made for ethical demands of practice, including issues of safety, prevention, and mandatory reporting. Questions, such as what a social service worker is responsible for if a client or group member expresses suicidal ideation while communicating via SL interactions and how to ensure that the client being treated is the client behind the avatar, must be given the utmost consideration before beginning this potential venture. These questions are likely to produce opinions and concerns across the spectrum of professional human services and will need to be defined ethically as well as legally.

\section{Conclusions}

This study represents the emerging use of virtual reality technology in the study of why a select sample of persons sought SL, what activities appealed to them, and what benefits were derived for them. The study was completed within the context of a graduate social work research course and applies an extended application of distance education to human behavior in a social environment. Data results indicate that 
interests of the individual in SL may to some degree reflect the interests they enjoy or once enjoyed in RL (due to a disability, for instance), making SL an opportunity for them to re-experience interests or accomplishments. Understanding this, SL has the potential to provide role plays for clients to enhance their RL social groups, communities, activities, and experiences. The methods utilized in this study can be directly applied to numerous research endeavors in using immersion in virtual worlds, such as the application of the social network knowledge construction (Dawley, 2009) within social service educational curriculum design. 


\section{References}

Anderson, T., \&Dron, J . (2011). Three generations of distance education pedagogy. The International Review of Research in Open and Distance Learning, 12(3), 8097. Retrieved from www.irodl.org/index.php/irrodl/article/download/890/ 1826.

Anstadt, S., Burnette, A., \& Bradley, S. (2011). Towards a research agenda for social work practice in virtual worlds. Advances in Social Work, 12(2), 289-300.

Anstadt, S., Burnette, A., \& Bradley, S. (2012). Advanced technology education: A case for uses of virtual reality in teaching human services. J ournal of Virtual Studies, 3(1), 264-273.

Aurilio, S. (2010). Learning in the wild of a virtual world (Doctoral dissertation). Retrieved from ProQuest Dissertations and Theses. (Accession Order No. [3399141]).

Baker, S., Wentz, R. K. \&Woods, M. M. (2009). Using virtual worlds in education: Second Life ${ }^{\circledR}$ as an educational tool. Teaching of Psychology, 36(1), 59-64. doi: 10.1080/00986280802529079

Barab, S., Thomas, M., Dodge, T., Carteaux, R., \& Tuzun, H. (2005). Making learning fun: Quest Atlantis, a game without guns. Educational Technology Research \& Development, 53(1), 86-107.

Belisle, J . F., \&Bodur, H. O. (2010). Avatars as information: Perception of consumers based on their avatars in virtual worlds. Psychology and Marketing, 27(8), 741765. doi: 10.1002/mar.20354

Beltran, L. M., Sierra, R. S., Gutierrez, C. \& Garzon-Castro, L. (2012). Second Life as a support element for learning electronic related subjects: A real case. Computers and Education, 58(1), 291-302. doi : http://dx.doi.org/ 10.1016/j.compedu.2011.07.019.

Bell, M. W., Castranova, E., \&Wagner, G. G. (2009). Surveying the virtual world: A large scale survey in SL using the Virtual Data Collection Interface (VCMI). Data Documentation, 44, 1-61.

Boellstorff, T., Nardi, B., Pearce, P. \& Taylor, T.L. (2012). Ethnography and virtual worlds: A handbook of method. Princeton: Princeton University Press.

Boellstorff, T. (2008). Coming of age in Second Life : An anthropologist explores the virtual human. Princeton: Princeton University Press. 
Brooks, M. (2002). Drawing to learn (Unpublished doctoral thesis). University of Alberta, Edmonton, AB, Canada.

Bruckman, A. (1997). MOOSE Crossing: Construction, community, and learning in a networked virtual world for kids (Unpublished PhD). MIT.

Burger, P. L., \&Luckmann, T. (1966). The social construct of reality: A treatise in the sociology of knowledge. Garden City, NY : Anchor Press.

Conners, K. A. (2009). A second life by design: Exploration of learning theory (Unpublished master's thesis). Hamline University, Saint Paul, Minnesota. Retrieved from www.hamline.edu/WorkArea/ DownloadAsset.aspx?id=2147491167

Damianakis, T., Climans, R., \& Marziali, E. (2008). Social workers' experiences of virtual psychotherapeutic caregivers groups for Alzheimers', Parkinson's, stroke, frontotemporal dementia, and traumatic brain injury. Social Work with Groups, 31(2), 99-115.

Dawley, L. (2009). Social network knowledge construction: Emerging virtual world pedagogy. On The Horizon, 17(2), 109-121. doi : 10.1108/10748120910910965494.

Delucia, A., Francese, R., Passero, I., \& Tortora, G. (2009). Development and evaluation of a virtual campus on Second Life: The case of Second DMI. Computers \& Education, 52(1), 220-233.

Dillenbourg, P., Schneider,D., \& Synteta,V.(2002). Virtual learning environments. Proceedings of the 3rd congress on information and communication technologies in education (pp. 3-18). Rhodes : Greece : Kastaniotis Editions.

Eastwick, P. W., \& Gardner, W. L. (2009). Is it a game? Evidence for social influence in the virtual world. Social Influence, 4(1), 18-32. doi:

$10.1080 / 15534510802254087$

Edwards, M.T. (2012). Tranformative learning theory as a framework for designing experiences in virtual worlds as appropriate to counsellor education. Retrieved from www.inter-disciplinary.net/wpcontent/ uploads/ 2012/ 02/ edwardsepaper.pdf

Erlandson, B. E., Nelson, B. C., \&Wilhelmina, C. S. (2010). Collaboration modality, cognitive load, and science inquiry learning in virtual inquiry environments. Educational Technology Research \& Development, 58(6), 693-710. 
Fetscherin, M., \& Lattemann, C. (2008). User acceptance of virtual worlds. J ournal of Electronic Commerce Research, 9(3), 231-242.

Gottschalk, S. (2010). The presentation of avatars in Second Life: Self and interaction in social virtual spaces. Symbolic Interaction, 33(4), 501-525. doi:

10.1525/ si.2010.33.4.501

Holloway, S., Black, P., Hoffman, K., \& Pierce, D. (n.d.). Some considerations of the import of the 2008 EPAS for curriculum design. Council on Social Work Education. Retrieved from http:// www.cswe.org/Accreditation/EPASImplementation.aspx

Huang, H.M. (2012). Toward constructivism for adult learners in online learning environments. British J ournal of Educational Technology, 33(1), 27-37.

Isabella, S. (2007). Ethnography of online role-playing games: The role of virtual and real contest in the construction of the field. Forum: Qualitative Social Research, 8(3), 1-16.

J armon, L., Traphagan, T., Mayrath, M., \&Trivedi, A. (2009). Virtual world teaching, experiential learning, and assessment: An interdisciplinary communication course in Second Life. Computers \& Education, 53(1), 169-182. doi:10.1016/j.compedu.2009.01.010

J ha, A. K. (2012). Epistomological and pedagogical concerns of constructionism: Relating to the educational practices. Creative Education, 3(2), 171-178. doi: 10.4236/ce.2012.32027.

Leonard, L., Withers, L. A., \& Sherblom, J . C. (2011). Collaborating virtually: Using "second life" to teach collaboration. Communication Teacher, 25(1), 42-47.

McCarty, D., \&Clancy, C. (2002). Telehealth: Implications for social work practice. Social Work, 47(2), 153-161.

Mills, H. (2012). Avatar creation: The social construction of "beauty" in second life (Unpublished master's thesis). The University of Akron, Akron, Ohio.

Minocha, S., \& Roberts, D. (2008). Laying the groundwork for solialisation and knowledge construction within 3D virtual worlds. ALT-J , Research in Learning Technology, 16(3), 181-196. doi: 10.1080/09687760802526699.

Mutekwe, E., Ndofirepi, A., Maphosa, C., Wadesango, N., \&Machingambi, S. (2013). A swot analysis of the rise and pedagogical implications of the social constructivist epistemology in educational practice. Anthrpologist, 15(1), 53-65. 
Nesson, R., \&Nesson, C. (2008). The case for education in virtual worlds. Sage Publications, 11(3), 273-284. doi: 10.1177/ 1206331208319149

Newman, T., Olle, M., \& Bradley, C. (2012). Social interaction as a contributor to significant learning outcomes in online instruction. International J ournal of Instructional Technology and Distance Learning, 9(5), 59-64.

Parti, K. (2008). Deviances in the virtual reality or the character-altering power of virtual communities. European J ournal of Crime, Criminal Law \& Criminal J ustice, 16(3), 325-343. doi:10.1163/ 157181708X33337

Pinkett, R. D. (2000). Constructionist learning in communities (Written component of Ph.D. general examination). Massachusetts Institute of Technology, Cambridge, Massachusetts.

Riedl, R. E., Tashner, J . H., \& Bronack, S. C. (2003). A virtual world initiative: Assumptions about teaching and learning. International Conference on New Educational Environments. Lucerne, Switzerland.

Rockinson-Szapkiw, A. J ., \& Walker, V. L. (2009). Web 2.0 technologies: Facilitating interaction in an online human services counseling skills course. J ournal of Technology in Human Services, 27, 175-193. doi:

10.1080/15228830903093031

Salmon, G., Nie, M., \&Edirisingha, P. (2010). Developing a five-stage model of learning in second life. Educational Research, Special Issue: Virtual Worlds and Education, 52(2), 169-182. doi:10.1080/ 00131881.2010.482744

Shaw, A.C. (1995). Social construction and the inner city: Designing environments for social development and urban renewal (Unpublished doctoral dissertation). MIT Media Laboratory, Cambridge, Massachusetts.

Smokowski, P. R., \& Hartung, K. (2003). Computer simulation and virtual reality: enhancing the practice of school social work. J ournal of Technology in Human Services, 21(1/2), 5-30.

Squire, K. \&J enkins, H. (2004). Harnessing the power of games in education. Insight, 3(1), 5-33.

Stalker, G.J . (2007). Measuring diversity in daily social contact: The contribution of social context, work and leisure on the opportunity for engagement. Springer Science+Buisness Media, 86, 275-295. doi: 10.1007/s11205-007-9115-5 
SparkNotes Editors (2006). Sparknote on identity and reality. Retrieved from http:// www.sparknotes.com/ sociology/ identity-andreality/ section1.rhtml

Sun, Y. (2012). Analysis and research on distance learning in second life. IPCSIT, 53. doi: 10.7763/IPCSIT.2012.V53.No.2.22.

Tsan, J . Y., \& Day, S. X. (2007). Personality and gender as predictors of online counseling use. J ournal of Technology in Human Services, 25(3), 39-55.

Vernon, R. , Lewis, L., \& Lynch, D. (2009). Virtual worlds and social work education: Potentials for "Second Life." Advances in Social Work, 10(2), 176-192.

Vygotsky, L.S., (1978). Mind in society. Cambridge, MA: Harvard University Press.

Wood, D. (2009). Real life access to "Second Life" worlds: The potential, the problems and the possibilities for a barrier-free future. The International J ournal of Diversity in Organizations, Communities, and Nations, 8(6), 139-148. 


\section{Appendix A}

SL Questionnaire

Directions: Please indicate any questions for which you feel uncomfortable in responding in a truthful manner.

1. Did you read the CONSENT FORM located on the screen in front of this kiosk?

a) Yes

b) No

2. If no, reading the consent is an important part of participating in this study. Please click "Exit Survey" and read the consent form before taking this survey.

a) Exit Survey

3. Do you agree to continue past this point and take the following survey?

a) Yes

b) No

c) Exit Survey

Demographics:

4. What is your $\mathrm{RL}$ age?
a) Age $18-30$
b) Age 30-50
c) Age 50-65
d) Age 65+
e) No response
f) Exit Survey

5.What is your ethnic background?

6. Gender
a. Male
b. Female
c. Other
d. No response
e. Exit Survey

7. What is your highest education level?

a. Some high school

b. High school graduate 

c. Some college
d. College graduate
e. Associate's Degree
f. Technical Degree
g. Master's Degree
h. Doctoral degree
i. No response
j. Exit Survey

8. Marital status?
a. Single
b. Divorced
c. Widowed
d. Engaged
e. Married (1-5yrs)
f. Married (5-15 yrs)
g. Married (15- beyond)
h. No response
i. Exit Survey

9. What country are you currently living in ?

10. How did you first hear about SL

11. What motivated you to join SL ? ?

12. How long have you been on SL ?

13. Is this your first Avatar in SL ?
a) Yes
b) No
c) exit survey

14. How many Avatars (Alts) do currently you have in SL ?
a) 1
b) 2
c) 3
d) 4
e) 5
f) 6
g) 7
h) More than 7
i) No response
j) Exit Survey 
15.Gender of Avatar(s)?
a) Male
b) Female
c) Other
d) No response
e) Exit Survey

16. How frequently do you sign into SL ?
a. Less than three times per week
b. 1 time per day
c. 2-4 times per day
d. 5-7 times per day
e. more than 7 interactions per day or stay signed on
f. No response
g. Exit Survey

17. How many hours are you in SL ?
a. Less than one hour per week
b. 1-4 hours per week
c. 5-9 hours per week
d. 10-20 hours per week
e. More than 20 hours per week
f. No response
g. Exit Survey

SL Experience:

18. Which of these choices do you consider to be most prominent for you in SL?:
a. Social Networking
b. An extension of RL
c. Gaming
d. Creating/ Displaying
e. Revenue
f. A combination of the above
g. No response
h. Exit Survey

19. Did you find SL difficult to navigate?
a. Yes
b. No
c. Exit survey

20. When you meet people in SL do you believe:

a. The avatar represents the real person. 
b. There is a low likelihood the avatar represents the real person.

c. The avatar is a contact to further my goals.

d. I do not relate to other Avatars

e. Exit Survey

21. Do you believe:

a. It is impossible to believe anything said online.

b. You can learn more about people behind the anonymity of the virtual.

e. For the reasons I am in SL, truth expression about RL doesn't matter

d. Other

e. Exit Survey

22. Do you feel you could make valuable, trustworthy friends in a virtual world?
a. Yes, absolutely!
b. Yes, sometimes
c. I'm not in SL to make personal connections
d. Strictly for role-play/ fantasy/gaming
e. No comment at this time
f. Exit Survey

23. Have you been dancing in SL ? Do you find it:
a. Yes, no further comment.
b. Yes, a good way to meet people and fun.
c. Yes, stimulating when the avatars are all synchronized.
d. Yes, better than avatars just standing around.
e. No, I'm here for other reasons.
f. No comment or opinion at this time.
g. Exit Survey

24. Have you done any role-playing (fantasy, space historical, Gorean, BDSM, etc) in SL?
a. Yes, no further comment.
b. Yes, all of SL is role play.
c. No, not interested.
d. No, I'm here for other reasons.
e. No comment.
f. Exit Survey

SL and RL Interaction:

25. Are you in an intimate/ romantic relationship in SL ?
a) Yes
b) No
c) Exit survey

26. How long have you been in this relationship? Please indicate how long? 
a) Less than six months

b) More than six months

27. Are any of your close friends or family regularly active on SL ?
a) Yes
b) No
c) Exit survey

28. How many socially interactive everyday contacts do you make in $\mathrm{RL}$ ?
a) less than 3 times per week
b) 1 interaction per day
c) 2-4 interactions per day
d) 5-7 interactions per day
e) more than 7 interactions per day
f) No response
g) Exit Survey

29. How many interpersonal social events do you average per day, with people close to you (i.e. close friends, family, lovers, etc.)
a. 1 interaction per day
b. 2-4 interactions per day
c. 5-7 interactions per day
d. More than 7 interactions per day
e. No response
f. Exit Survey

30. Do you get personal satisfaction from interacting with people in $R L$ ? $\begin{array}{lllllllll}\text { Not at all } & 1 & 2 & 3 & 4 & 5 & 6 & 7 & \text { All the time }\end{array}$

31. What motivates you to attend group or community meetings in SL ?
a. Friends
b. Education
c. Business
d. Social Support
e. Other
f. No response
g. Exit Survey

32. How many planned, interpersonal social contacts do you have in SL per day?
a. Less than 3 per week
b. 1 per day
c. 2-4 per day
d. 5-7 per day
e. 7 or more per day 


\section{f. No response}

g. Exit Survey

33. Do you get personal satisfaction from interacting with others in SL ? Please select an answer from 0 to 7 where 0 is Not at All and 7 is All the Time.

$\begin{array}{lllllllll}\text { Not at all } & 1 & 2 & 3 & 4 & 5 & 6 & 7 & \text { All the time. }\end{array}$

34. Pick one activity in SL that you find to be the most motivating (meaning you want to do it again) and list it here

35. Do you find the activity in the previous question motivating in a similar way in RL?
a. Yes
b. No
c. Partially
d. Exit survey

36. Have you ever met a person in $\mathrm{RL}$ that you first met in SL ?
a. Yes
b. No
c. Planning to
d. Exit survey

37. Was the meeting a positive experience?
a. Yes
b. No
c. Neutral
d. Exit survey

38. Would you like to help us further by agreeing to meet with one of the researchers for a brief 6 question interview at your convenience?
a) Yes
b) No

If yes, Thank you! You will be given a notecard with your gift of a free tee at the end of this survey. Please open the box and enjoy your gift. Info will be provided if you change your mind about the interview, but you will not be contacted further.

If no, Thank you! You will be given a notecard with your gift of a free tee at the end of this survey. Please open the box and enjoy your gift. Info will be provided if you change your mind about the interview, but you won't be contacted.

All done! Thank you for participating in the survey! Please accept our gift of a free graphic tee! If you have any questions please feel free to contact the researchers (see notecard attached to your tee) for additional information. 


\section{Appendix B}

Qualitative Interview Consent and Questions

By answering the questions and e-mailing the researchers to schedule to meet in SL you are consenting to the interview portion of this study. Know that your answers to the above questions will be kept in confidence in the same way that your survey will be. The below questions will be the only questions asked during the interview, and we may only ask "Would you like to share any more information about that?" but only about the topics in the questions below. These questions and your survey will be kept separate and there will be no identifying information connected with your answers. Your username and/ or e-mail will be coded in that it will be given a number meaning that your answers in no way can be identified to you. The answers to these questions will be saved on a password protected USB in a locked office and in a locked drawer, accessible only to the faculty advisor and the researchers, and the information will be destroyed after 3 yrs.

Thank you for your time and consideration!

Interview Questions: SL

1. How did you hear about SL ? Please explain?

2. What made you want to join SL ? Once you did, was it easy to navigate and why?

3. What is your favorite thing to do in SL ? Why?

4. What do you perceive that you get out of being a member of SL ? Please explain?

5. Do you feel like you connect with individuals on SL ? Do you connect with individuals in RL ? (e.g. Friends, romantic relationships, groups)

6. If applicable list any groups you belong to in SL and/ or RL ? Why did you join?

These next few questions are optional:

7. Do you feel that your avatar is an accurate representation of your RL physical appearance? Please explain?

8. If you have multiple avatars, what is the purpose in having multiple? Please explain?

9. Do you feel that your time on SL fulfills needs that may not be met in RL ? Please explain? 


\section{Appendix C}

Table 1

Real Life and Second Life Demographics ( $\mathrm{N}=100)$

Dimension

Age in RL

Ethnicity

Gender in RL

Marital status in RL

Education
Attributes

18-30

31-50

51-65

Above 65

No Response

Caucasian

African American

Pacific Island

Hispanic

Other European

Other

No Response

Male

Female

Other

Single

Married 1-5years

Married 5-15 years

Married over 15 years

Divorce

Widowed

Engaged

Other

Some high school

High school grad.

Some college

College graduate

Associates degree

Technical degree

Masters degree

Doctorate

No response
Frequency

Percentage

18

43

29

8

2

77

1

5

3

10

2

2

38

59

3

18.4

43.9

29.6

8.2

2.0

78.6

1.0

5.1

3.1

10.2

2.0

2.0

38.0

59.0

3.0

28

28.0

13.0

10.0

29.0

12.0

3.0

3.0

2.0

1.0

2.0

21.2

23.2

7.1

1.0

31.3

13.1

1.0 


\begin{tabular}{llcc} 
& USA & 70 & 71.4 \\
\multirow{4}{*}{ Country of residence } & Europe & 17 & 17.3 \\
& Australia & 3 & 3.1 \\
& S. America & 2 & 2.0 \\
& Canada & 6 & 6.1 \\
& No response & 2 & 2.0 \\
& & & \\
Number of avatars & 1 & 37 & 37.0 \\
& 2 & 26 & 26.0 \\
& 3 & 15 & 15.0 \\
& 4 or more & 15 & 15.0 \\
Avatar gender & & & \\
& & & 37.0 \\
& Male & 37 & 59.0 \\
& Female & 59 & 4.0 \\
Other & 04 & \\
Avatar age & & & 15.0 \\
& & 15 & 9.0 \\
& Less than 3 months & 09 & 19.0 \\
& 3 months- 12 months & 19 & 7.0 \\
& 1-2 years & 49 & 1.0
\end{tabular}

\section{Athabasca University $\mathbf{Z}$}

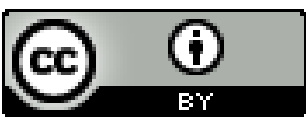

